\title{
The kiss of the girl from Ipanema
}

\author{
R. Evertz • J. Brugada
}

Published online: 3 July 2013

(C) The Author(s) 2013. This article is published with open access at Springerlink.com

\section{Answer to the rhythm puzzle}

The ECG shows a narrow QRS tachycardia, although without a very fast ventricular rate of 110 beats $/ \mathrm{min}$. A rather narrow $P$ wave can be appreciated just after the $\mathrm{T}$ wave with a positive deflection in leads V1 to V6, I and aVL and negative deflection in leads II, III and aVF. Another P wave can be found right at the end of the QRS complex, giving rise to a pseudo $\mathrm{R}$ wave in lead V1, or RSR ${ }^{1}$. This pseudo R wave is absent during sinus rhythm (Fig. 2a-c). An orthodromic atrioventricular reentrant tachycardia (AVRT) can be excluded, because the ventricle is not part of the reentry circuit. The differential diagnosis is either an atrioventricular nodal reentrant tachycardia (AVNRT) with 2:1 block to the ventricle or an atrial tachycardia (AT) with 2:1 atrioventricular (AV) block. The Pwave morphology in this case is not very helpful, because it suggests an activation pattern from inferior to superior and a septal origin, which can be seen in both an AVNRT and a posteroseptal focal AT. During the electrophysiology study, dual AV nodal physiology was observed and with programmed stimulation a typical AVNRT with a short AV interval and 1:1 AV relation was induced after two supraventricular extra stimuli with the second clearly conducted over the slow pathway (long AV interval with a jump) and retrograde conduction over the fast pathway. After repeated induction the same phenomenon as observed on the ECG of Fig. 1 was seen with 2:1 conduction block to the ventricle. This tachycardia started in the same manner as the one with 1:1 conduction and showed a fixed atrioatrial and ventriculoatrial interval. After radiofrequency ablation of the slow pathway at the posterior aspect of Koch's triangle no tachycardia could be induced. Looking back at the initial ECG we see a pattern that has been called 'the kiss of the girl from Ipanema' most clearly seen in lead aVL. This pattern is typical for an AVNRT with 2:1 conduction block to the ventricle and appears because of the position of the retrograde $P$ wave directly after the T wave ${ }^{1}$ (Fig. 2d). It was named after a song called 'The girl from Ipanema', written and first performed in Rio de Janeiro by Antonio Carlos Jobim in 1962. It was awarded a Grammy in 1964 for best song of the year. Thereafter it has been performed by many artists, including Frank Sinatra, Ella Fitzgerald and Madonna.

Open Access This article is distributed under the terms of the Creative Commons Attribution License which permits any use, distribution, and reproduction in any medium, provided the original author(s) and the source are credited.
R. Evertz $(\bowtie) \cdot J$. Brugada

Cardiology Department - Arrhythmia Section,

Thorax Institute - Hospital Clínic, University of Barcelona,

Villarroel 170,

08036 Barcelona, Spain

e-mail: reinderevertz@gmail.com 

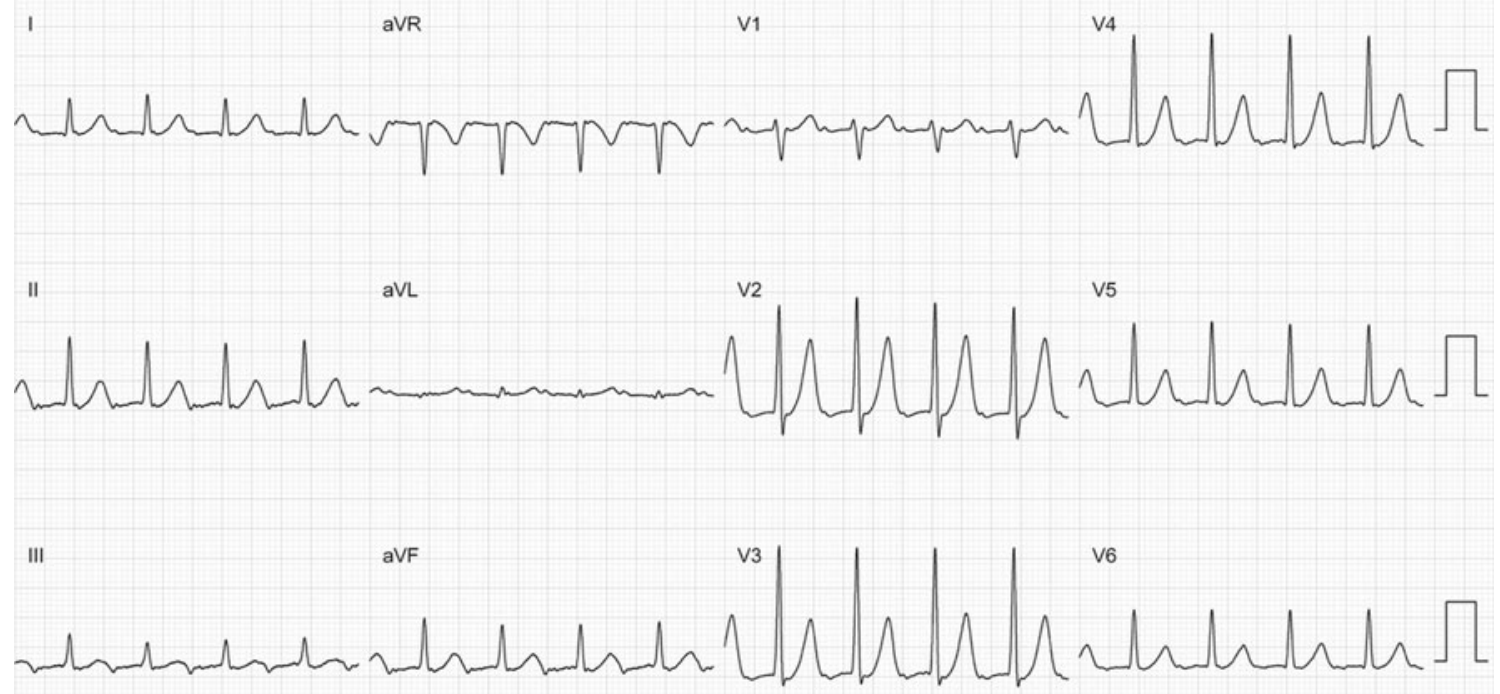

॥

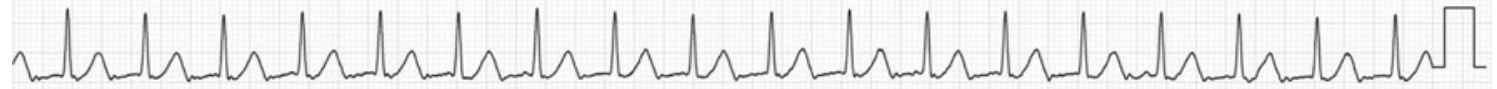

$25 \mathrm{~mm} / \mathrm{s} 10 \mathrm{~mm} / \mathrm{mv}$

Fig. 1 Twelve-lead surface ECG of the tachycardia 


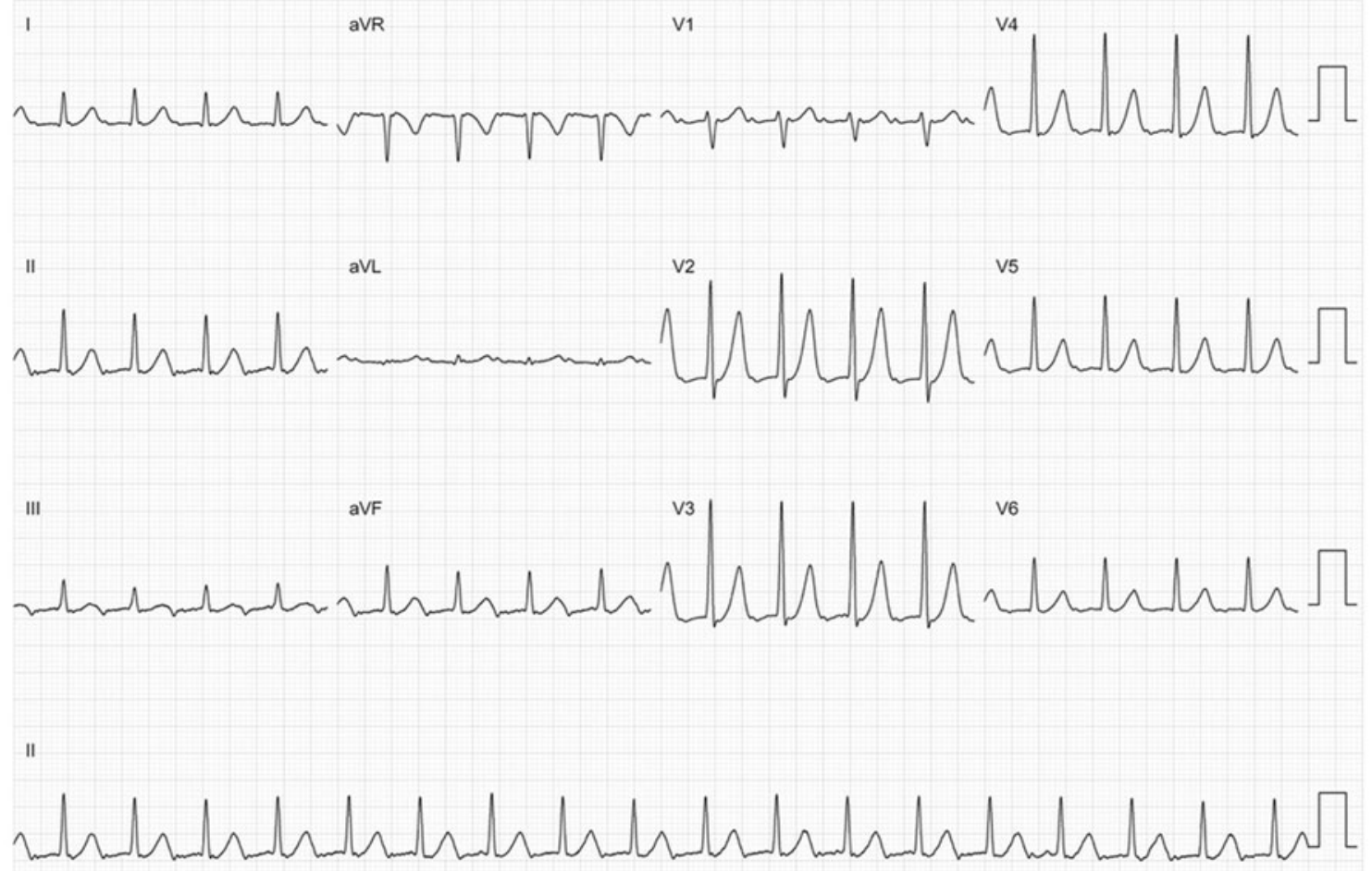

A

$25 \mathrm{~mm} / \mathrm{s} 10 \mathrm{~mm} / \mathrm{mv}$

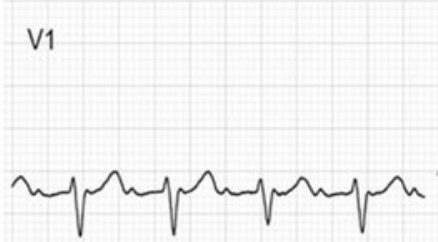

B

Fig. 2 a. The 12-lead ECG of the tachycardia with 2:1 conduction block to the ventricle. b. Lead V1 with a pseudo R-wave visible at the terminal portion of the QRS and the second $\mathrm{P}$ wave right after the $\mathrm{T}$

C
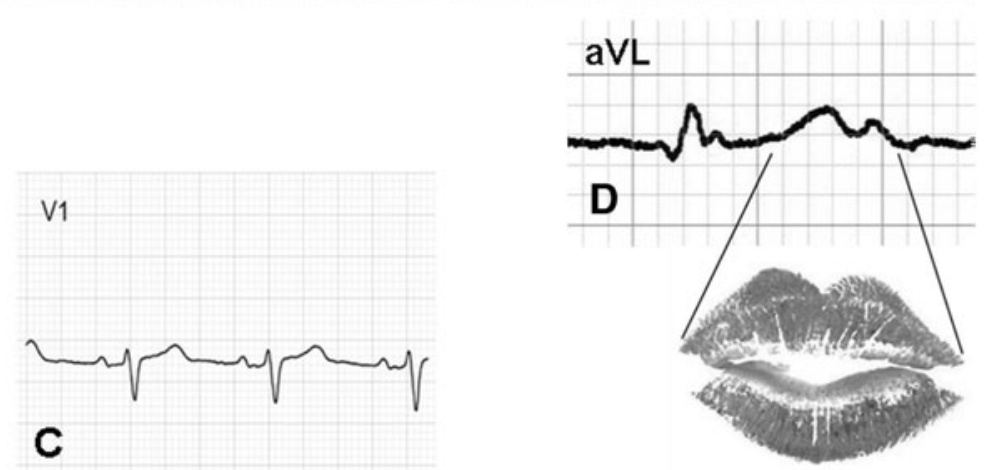

wave. c. Lead V1 of the same patient during sinus rhythm with absence of the pseudo $\mathrm{R}$ wave. d. Lead aVL magnified showing the pattern called: 'the kiss of the girl from Ipanema' 